\title{
CFD analysis and optimization of the sensor "MicroMED" for the ExoMars 2020 mission
}

\author{
Giuseppe Mongelluzzo ${ }^{1,2, *}$, Francesca Esposito ${ }^{1}$, Fabio Cozzolino ${ }^{1}$, Cesare Molfese ${ }^{1}$, Simone Silvestro ${ }^{1,3}$, Gabriele \\ Franzese $^{1}$, Ciprian Ionut Popa ${ }^{1}$, Marek Lubieniecki ${ }^{4}$, Fausto Cortecchia ${ }^{5}$, Bortolino Saggin ${ }^{6}$, Diego Scaccabarozzi ${ }^{6}$, \\ Alexander Zakharov ${ }^{7}$
}

${ }^{1}$ INAF - Osservatorio Astronomico di Capodimonte, Salita Moiariello, 16, 80131, Napoli, Italy

${ }^{2}$ Department of Industrial Engineering, University of Naples "Federico II"

${ }^{3}$ SETI Institute, Carl Sagan Center, Mountain View, California, USA

${ }^{4}$ Delft University of Technology, Postbus 5, 2600 AA Delft

${ }^{5}$ INAF - Osservatorio Astronomico di Bologna, Bologna Italy

${ }^{6}$ Department of Mechanical Engineering, Politecnico di Milano, Lecco, Italy

${ }^{7}$ IKI, Moscow, Russia

* Corresponding author, giuseppe.mongelluzzo@inaf.it

Full Postal address: Salita Moiariello, 16, 80131, Napoli, Italy

\begin{abstract}
Characterization of dust is a key aspect in recent space missions to Mars. Dust has a huge influence on the planet's global climate and it is always present in its atmosphere. MicroMED is an optical particle counter that will be part of the "Dust Complex" suite led by IKI in the ExoMars 2020 mission and it will determine size distribution and concentration of mineral grains suspended in martian atmosphere. A Computational Fluid Dynamic (CFD) analysis was performed aimed at the optimization of the instrument's sampling efficiency in the $0.4-20 \mu \mathrm{m}$ diameter range of the dust particles. The analysis allowed to understand which conditions are optimum for operations on Mars and to consequently optimize the instrument's fluid dynamic design.
\end{abstract}

Keywords-MicroMED; ExoMars 2020; CFD; Mars

\section{INTRODUCTION}

MicroMED is an optical particle counter designed to measure both size distribution and abundance of dust grains in martian atmosphere. Characterization of airborne dust is a pivotal aspect of modern martian missions, since dust deeply influences the planet's global climate, particularly during the dust storms season. The dynamics of dust and the possible generated electrical field [1-3] are currently focal points of Martian research and are fields that are going to be studied in detail during future Mars missions. MicroMED will be part of the Russian led "Dust Complex" suite onboard the ExoMars 2020 mission. It is a miniaturized and simplified version of the MEDUSA [4,5] instrument, developed at INAFAstronomical Observatory of Capodimonte in Naples, Italy, where the characterization of dust in Mars, Earth and Solar System environments has recently been the center of numerous studies (within the DREAMS [6-8], MEDUSA and GIADA [9-12] projects). Preliminary results of this study have been presented at the "Metrology for Aerospace 2018" Conference held in Rome (June 20-22, 2018) [13]. This paper furthermore evidences the Computational Fluid Dynamic (CFD) model setup and its validation by means of comparison with experimental tests. New evidence is presented in the results chapter outlining the expected performance of the instrument. 
MicroMED layout can be seen in Fig. 1-2. It has a sampling head, exposed to the Martian atmosphere, whose four inlet holes allow suction of gas from outside. The fluid is conveyed toward the sampling section by means of a conic duct. After the inlet there is a small gap between ducts allowing optical scan of the flow. The fluid dynamic design is conceived in order to make dust grains cross a $1 \mathrm{~mm}^{2}$ sampling spot where a laser beam is focused. Light scattering by dust grains is then analyzed as particles scatter light differently depending on their size. Scattered light is detected by a photodiode and the information is sent to the instrument's main electronics for signal amplification, conditioning, sampling and data processing. Dust concentration measurement can be provided because the volumetric flow rate at the inlet section is measured as well. After the gap, an outlet duct is connected to a pump allowing the expulsion of gas. The pump must provide the appropriate pressure difference between the outlet and inlet sections of MicroMED in order to achieve the required flowrate.

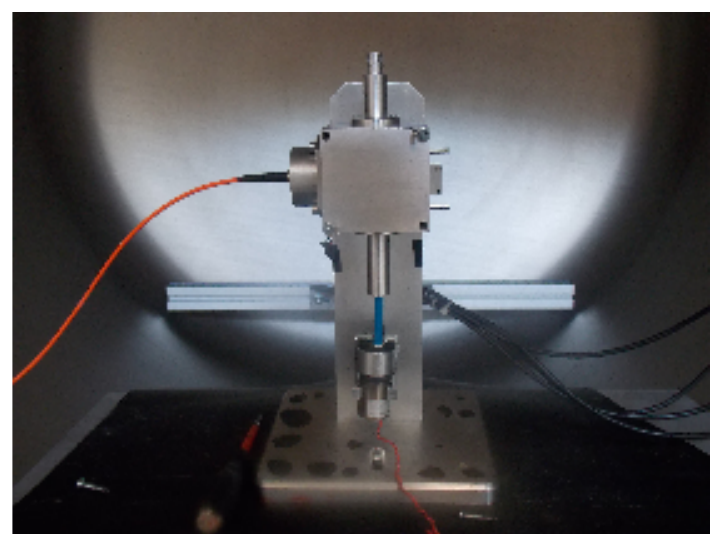

Fig. 1 MicroMED's Elegant Breadboard inside the Martian Simulation Chamber at the INAF-Astronomical Observatory of Capodimonte, Naples, Italy.

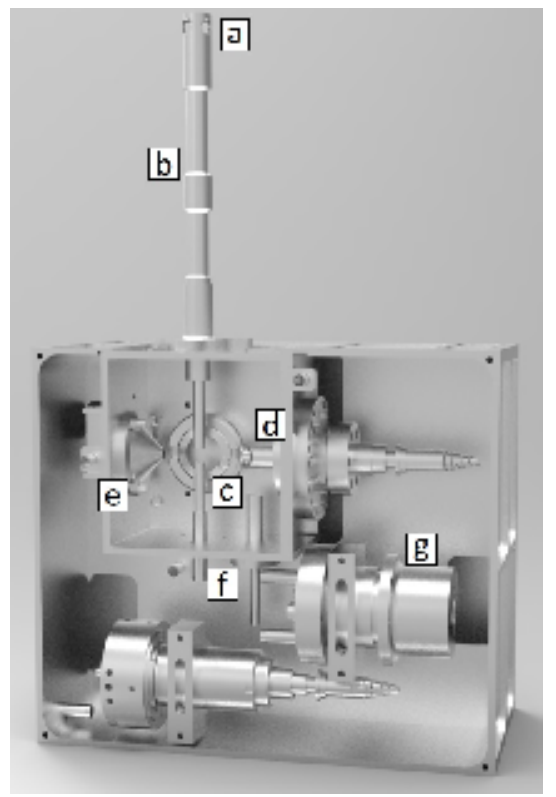

Fig. 2 MicroMED Proto Flight Model. (a) inlet head, (b) inlet duct, (c) sampling section, (d) laser emission system, (e) light trap (f) outlet duct, (g) pump

The instrument's feasibility was already verified in previous works [14], and its design is conceived in order to optimize the detection of suspended dust grains in the 0.4-20 $\mu \mathrm{m}$ diameter range. A number of possible issues related to MicroMED's fluid dynamics can affect the correct functioning. When the fluid sucked reaches the 4 $\mathrm{mm}$ gap between the ducts, it experiences an expansion that might deflect the dust grains' trajectories so that they do not cross the sampling spot. Indeed, the Stokes number of dust grains in the sampling range varies between $2 \cdot 10^{-}$ ${ }^{4}$ and 0.54 (four order of magnitude difference), thus dust grains at the extremes of the sampling range are expected to have different behavior. Another issue is the behavior of grains whose diameter is larger than $15 \mu \mathrm{m}$. Since they have high inertia, those particles tend to hit the walls while moving through the inlet duct because grains deflection is not swift enough. The inlet duct's mean surface roughness was considered approximately of the same order of magnitude as the grains diameter, meaning that dust grains colliding with the walls may get stuck, preventing their detection. In order to minimize the effect of those undesired phenomena, a detailed fluid dynamic analysis was needed to investigate which pump operating conditions optimize the instrument efficiency in terms of fraction of particles entering the instrument that actually passes through the sampling area. The analysis of the influence that environmental parameters and operating conditions have on the instrument's performance enabled 
to individuate criticalities and to develop an optimized design for the Flight Model.

Computational Fluid Dynamics modelling allowed to simulate the different environmental conditions the instrument might be exposed to, deriving information about how the instrument behavior changes while varying pump operating and environmental conditions. To facilitate the analysis, the "sampling efficiency" parameter was defined as the ratio of dust grains that are correctly detected by MicroMED to the total number of grains that cross the sampling spot. Indeed, during CFD simulations, dust grains that flow through the instrument can be tracked, determining the particles' trajectory. It can thus be determined whether dust grains' trajectory while crossing the sampling plane is inside the $1 \mathrm{~mm}^{2}$ spot, allowing the calculation of sampling efficiency.

Since a measurement of concentration is one of the goals of the instrument, information on the volumetric flow rate have to be obtained in order to relate the number of detected particles to the volume of sucked fluid.

\section{MICROMED CFD MODEL}

Simulations were set up considering the actual operating conditions of the instrument, which are meant to fulfill a number of requirements. First of all, MicroMED is focused on dust grains whose diameter ranges from 0.4 to $20 \mu \mathrm{m}$. Particles have to be conveyed toward a $1 \mathrm{~mm}^{2}$ area inside the instrument. Laminar flow condition is desired, as turbulent flow causes larger deflections of the particles trajectories and introduces an error due to the possibility of detecting some particles more than once. To avoid such error, the design has to guarantee a small fraction of coincidence $f($ e.g. $<0.05)$ in order to have a single particle counter and a large number of particles detected in a short time. Size distribution and volume density of dust grains must not alter measurements of size distribution and volume density. In addition, the optics must not be obstructed by the ducts walls. Therefore, mirrors do not have to be shadowed and the laser beam doesn't have to be intercepted by the instrument walls in any way.

\subsection{Environmental conditions}

CFD runs were performed simulating the different environmental conditions the instrument might face during the execution of the operations on Mars surface. The goal was to understand the evolution of key fluid dynamic parameters (sampling efficiency and inlet volumetric flow rate above all) while variating environmental conditions. Thus, in our simulations atmospheric pressure, atmospheric temperature and instrument temperature values were set in agreement with those predicted for the landing site (Oxia Planum) by the Mars Climate Database (MCD) [15] and from instrument thermal analysis. Overall, atmospheric pressure varies seasonally on Mars [16-17]. Three different values (600, 700 and $800 \mathrm{~Pa}$ ) were simulated in relation to the pressure annual variability. Daily variations were also taken into account during the simulation campaign. The highest variation according to the aforementioned model was 38 $\mathrm{Pa}$ and this was the value considered during the analysis. Three different atmospheric temperatures (190, 230 and $280 \mathrm{~K}$ ) were assumed for the runs. Since MicroMED will be under a thermal cover while on the lander, in our run we limited temperature variations to the $253-313 \mathrm{~K}$ range. Therefore, in first place "cold instrument" $\left(\mathrm{T}_{\mathrm{i}}=253 \mathrm{~K}\right)$ and "hot instrument" $\left(\mathrm{T}_{\mathrm{i}}=313 \mathrm{~K}\right)$ scenarios were simulated, considering the two extreme values of the interval, then runs for 5 additional instrument temperatures were added in order to complete the analysis. Eight different pump operating conditions were taken into account, related to an inlet-outlet pressure difference between 250 and 500 $\mathrm{Pa}$. Simulations used $\mathrm{CO}_{2}$ as fluid since it constitutes $95.3 \%$ of Martian atmosphere, but runs with air were also executed in order to validate the model with ground tests. Tab. 1 synthesizes the input parameters used for simulations.

\begin{tabular}{|c|c|c|c|}
\hline Parameter & $\begin{array}{c}\text { Min } \\
\text { value }\end{array}$ & $\begin{array}{c}\text { Max } \\
\text { value }\end{array}$ & $\begin{array}{c}\text { Number of } \\
\text { different } \\
\text { values }\end{array}$ \\
\hline $\mathrm{p}_{\mathrm{a}}$ & $600 \mathrm{~Pa}$ & $800 \mathrm{~Pa}$ & 3 \\
\hline $\mathrm{T}_{\mathrm{a}}$ & $190 \mathrm{~K}$ & $280 \mathrm{~K}$ & 5 \\
\hline $\mathrm{T}_{\mathrm{i}}$ & $253 \mathrm{~K}$ & $313 \mathrm{~K}$ & 8 \\
\hline$\Delta \mathrm{p}$ & $250 \mathrm{~Pa}$ & $500 \mathrm{~Pa}$ & 8 \\
\hline \multicolumn{3}{|c}{ Tab. 1 Input parameters for runs } \\
\hline
\end{tabular}

\subsection{CFD runs setup}

In order to verify that a Navier-Stokes based solver can be used for the analysis, the Knudsen number for the instrument had to be calculated. The reference length used is the minimum diameter of the instrument ducts $(1 \mathrm{~mm})$. As for the mean free path, it is dependent on the gas composition, temperature and pressure. Given that the Maxwell velocity distribution for gas molecules is applicable, the mean free path can be calculated using the formula [18]: 


$$
l=\frac{k_{B} T}{\sqrt{2} \pi d_{M}^{2} p}
$$

Where $k_{B}$ is the Boltzmann constant, $T$ and $p$ are the gas temperature and pressure and $\mathrm{d}_{\mathrm{M}}$ is the molecules diameter $\left(\mathrm{CO}_{2}\right.$ has been considered $)$.

In the cases of interest of MicroMED, $\mathrm{Kn}$ is always under 0.007 meaning the continuum assumption was always valid (it is considered valid if Knudsen number is smaller than 0.1 ). CFD code "Fluent" ${ }^{\circledR}$ by Ansys, in its version v18.1, was thus utilized for the analysis. The peculiar conditions of Mars atmosphere and the velocity fields experienced in the analysis allowed to use laminar model for runs. Indeed, given that both the reference length used (the same of $\mathrm{Kn}$ ) and the martian atmosphere density are very low, even for the highest values of fluid speed found in the simulations the Reynolds number was always under 1000. Anyway, CFD runs considering possible turbulent transition and wall effects were performed using the SST Transition model. The results of such runs confirmedthe assumption of laminar flow inside the instrument.

Particles were simulated as spherical and their drag law was customized by means of a user defined function considering Cunningham correction factor for low Reynolds number flows. Indeed, the Knudsen number related to the dust grains has been calculated and its value ranges, for the dust grains of MicroMED's sampling range, between 0.67 and 33.45, showing the need for a correction factor. The Cunningham correction factor was considered good enough given that dust grains have $\mathrm{Kn}_{\mathrm{P}}$ $\leq 1$ for part of the sampling range and that for $\mathrm{Kn}_{\mathrm{P}}<500$ there is plenty of examples in literature about how to accurately derive the experimental parameters needed [19-21]. The suction of fluid was simulated setting the atmospheric pressure value in proximity of MicroMED's head (on a hemispheric surface around the inlet's head that represents the simulated volume's border) and at the pump's inlet section, with suction caused by the pressure difference between these two regions of the simulated volume.

\subsection{Validation of the model}

In order to evaluate the quality of the model, CFD simulations were compared with tests performed on the instrument's Elegant Breadboard in the Martian Simulation Chamber located at the INAF - Astronomical Observatory of Capodimonte in Naples, Italy. The chamber allows to simulate martian conditions in terms of ambient pressure (and possibly composition). In particular, average values of dust grain speed in the sampling spot (a key fluid dynamic parameter) were used for comparison with the simulated dust grain speed. A simulations set using air as gas and was performed at ambient conditions given that some preliminary tests were performed in laboratory conditions. Tests used different monodispersed grains samples of $\mathrm{SiO}_{2}$ (with three different diameters of 1, 4 and 10 microns) and the comparison shows there is a good match between CFD and experimental results (Tab. 2). Indeed, values obtained from CFD are within the range of the average value obtained by tests for the $10 \mu \mathrm{m}$ grains (for which we have a smaller sample though, so a less reliable statistic) while, for 1 and $4 \mu \mathrm{m}$ ones, there are $2 \%$ and $8 \%$ differences on the average value, respectively. Given that such variations on the dust grains speed don't affect their dynamics inside the instrument, those values are good for our purposes, therefore the model can be considered valid. Fig. 3-5 report velocity histograms for the three aforementioned dust grains size derived from tests.

\begin{tabular}{|c|c|c|}
\hline Size $[\boldsymbol{\mu m}]$ & CFD & Tests \\
\hline 1 & $21.0 \pm 0.1$ & $21.5 \pm 0.1$ \\
\hline 4 & $20.4 \pm 0.1$ & $22.1 \pm 0.2$ \\
\hline 10 & $16.0 \pm 0.3$ & $16.5 \pm 0.7$ \\
\hline
\end{tabular}

Tab. 2 Comparison between average values of dust grains speed in sampling section obtained by CFD and tests

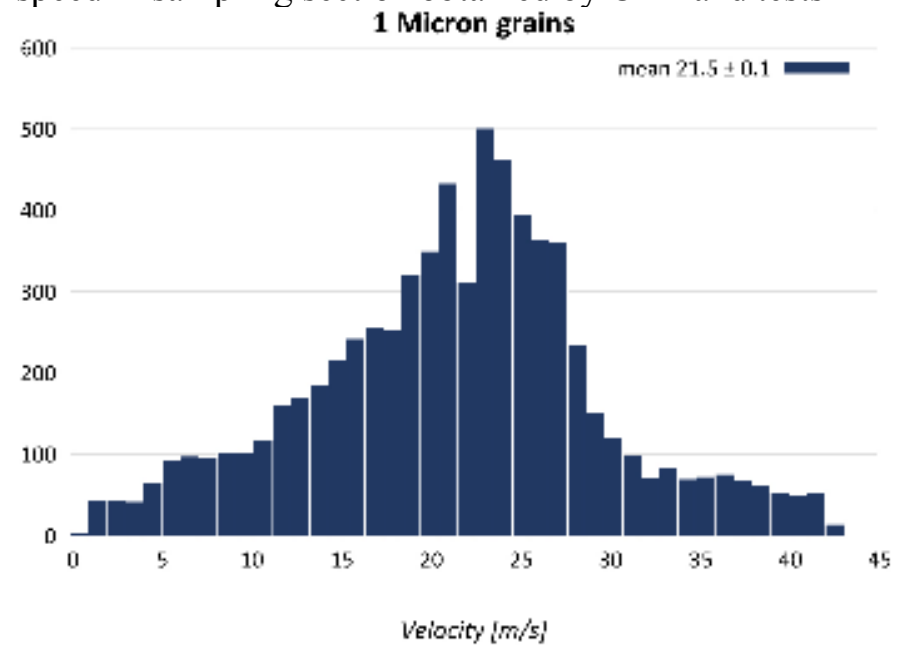

Fig. 3 Velocity histogram derived from tests for $1 \mu \mathrm{m}$ monodispersed particles 


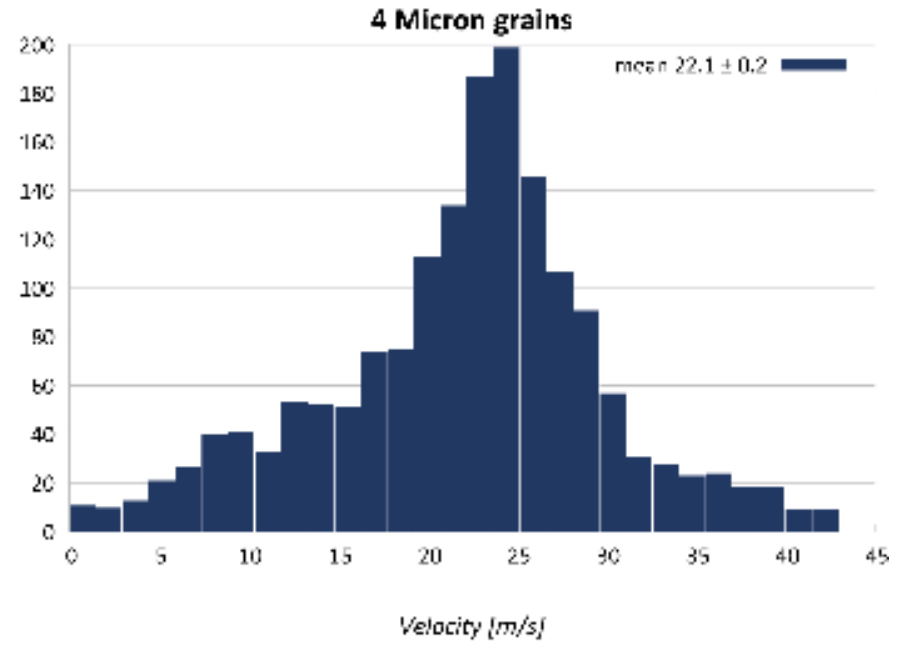

Fig. 4 Velocity histogram derived from tests for $4 \mu \mathrm{m}$ monodispersed particles

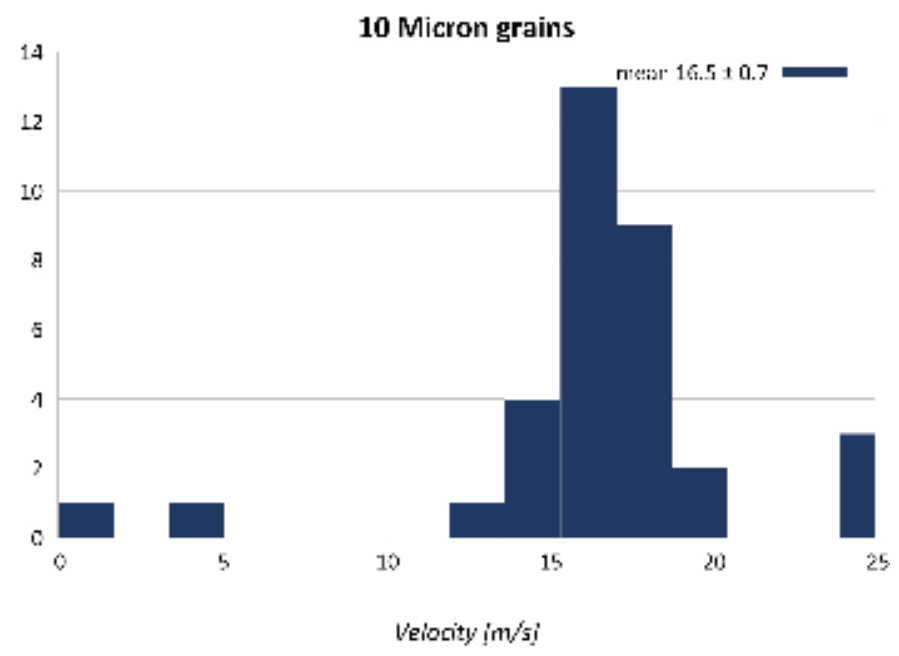

Fig. 5 Velocity histogram derived from tests for $10 \mu \mathrm{m}$ monodispersed particles

\section{SimULATIONS RESULTS}

\subsection{Sampling Efficiency}

Results show different behaviors for small $(0.4-1 \mu \mathrm{m}$ in diameter), intermediate $(1-15 \mu \mathrm{m})$ and large $(15-20 \mu \mathrm{m})$ particles. Grains of intermediate dimensions are always well detected as sampling efficiency values range from $95 \%$ to $99.5 \%$. On the other hand, the instrument ability to detect smaller and bigger grains changes with environmental parameters, so the instrument is able to optimize the efficiency of the instrument for the entire 0.4-20 $\mu \mathrm{m}$ diameter range only in the optimum operating conditions described hereafter. The following paragraphs will in fact describe the effect of the main environmental parameters on the instrument's sampling efficiency and discuss the derived optimum condition.

\subsubsection{Influence of instrument temperature on Sampling Efficiency}

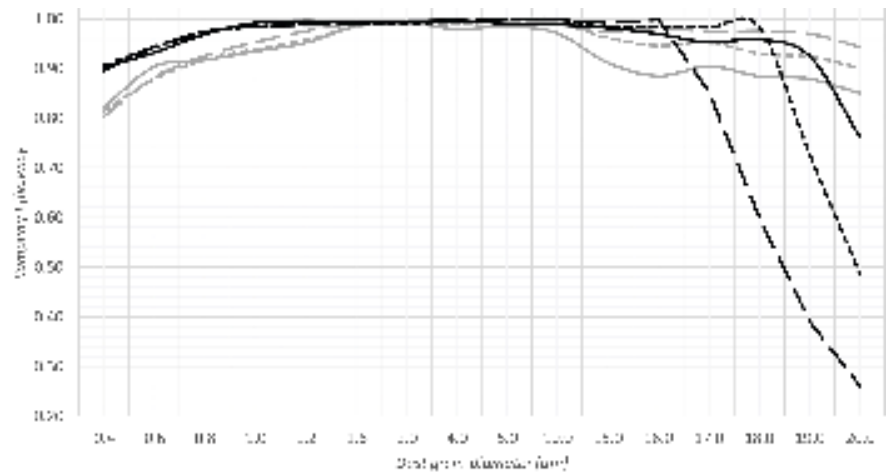

Fig. 6 Effect of instrument temperature on the instrument sampling efficiency. Plots are relative to $\mathrm{p}_{\mathrm{a}}=700 \mathrm{~Pa}$, $\Delta \mathrm{p}=300$ Pa. BLACK: $\mathrm{T}_{\mathrm{i}}=253 \mathrm{~K}$, GREY: $\mathrm{T}_{\mathrm{i}}=313 \mathrm{~K}$.

Instrument temperature is the most influential parameter. As we can see in Fig. 6, very different results are obtained for "cold instrument" runs (having $\mathrm{T}_{\mathrm{i}}=253 \mathrm{~K}$ ) and "hot instrument" runs (having $\mathrm{T}_{\mathrm{i}}=313 \mathrm{~K}$ ). Detection of small grains is more accurate for "cold" instrument (efficiency improvements up to $8 \%$ ) and for low pump rpm speed (generating an inlet-outlet pressure difference of $250 \mathrm{~Pa}$, the minimum value considered). When pump rpm speed increases, hot and cold runs show similar results for small grains. On the other hand, the detection of big dust grains (15-20 $\mu \mathrm{m})$ might be more problematic since high pump speeds (needed for better sucking ability) increase the chances of hits on the walls. Consequently, some of those particles might become undetectable. When the instrument is cold this chance heavily increases, probably because fluid inside the instrument is denser thus it offers more resistance to the deflection of grains inside the instrument's head, enhancing the chances of impact. This efficiency drop is critical and it was one of the main focuses of the present study. An improvement to the instrument design was developed based on these results in order to avoid such behavior without altering other key features of the instrument. For instance, the ability to have a high volumetric flow rate must be maintained (it might be reduced by changing of inlet and/or outlet geometry, worsening the instrument ability to suck fluid) and other fluid dynamic properties also have to be retained (different geometry may reduce the fluid speed inside the instrument, so deflection of dust grains outside the sampling spot might happen with higher probability). 
The new design has been analyzed and preliminary results appear to be very positive (Fig. 7).

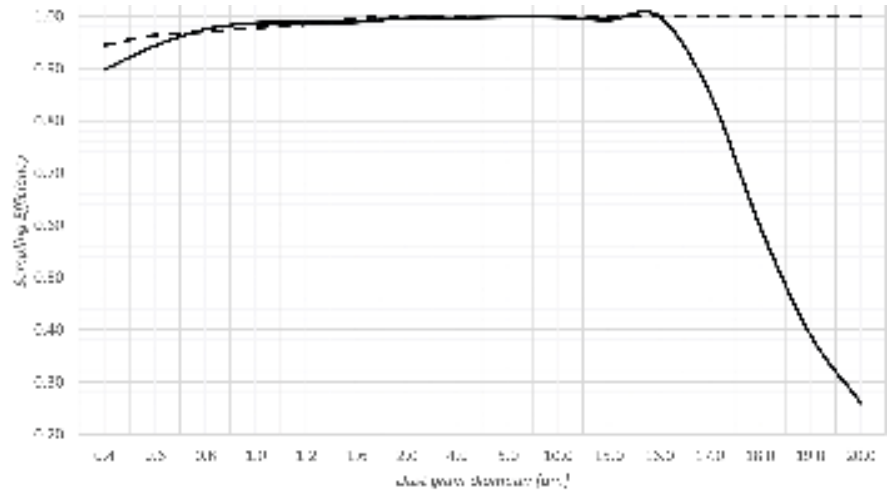

Fig. 7 Comparison between breadboard geometry and the newly designed geometry, $\mathrm{p}_{\mathrm{a}}=700 \mathrm{~Pa}, \mathrm{~T}_{\mathrm{i}}=280 \mathrm{~K}, \mathrm{~T}_{\mathrm{a}}=253$ $\mathrm{K}, \Delta \mathrm{p}=300 \mathrm{~Pa}$.

\subsubsection{Influence of atmospheric pressure on sampling efficiency}

As already stated, Mars' atmospheric pressure follows seasonal behavior. According to the MCD Model [15] daily variations are small compared to the absolute value of ambient pressure and simulations showed they have little influence on sampling efficiency (Fig. 8). Therefore, the seasonal value of pressure is the only variable. However, its value can be considered constant during the single run giving the short duration of the measurement session (that lasts about 120 seconds, with 30 seconds devoted to warm-up and self-check and 90 seconds of scientific measurement). Consequently, it was not considered necessary to install an absolute pressure sensor on MicroMED, an assumption confirmed by the fact that even seasonal atmospheric pressure values have small effects on the instrument ability to suck and detect particles (Fig. 9). This probably happens because the changes in the atmosphere physical parameters (viscosity and density) are not relevant to the point of changing the shape of the fluid stream. On the other hand, a differential pressure sensor is needed in order to achieve the proper functioning of MicroMED, since the pressure difference between the atmosphere and the outlet section of the instrument allows to evaluate and control the volumetric flow rate and understand the actual operating conditions.

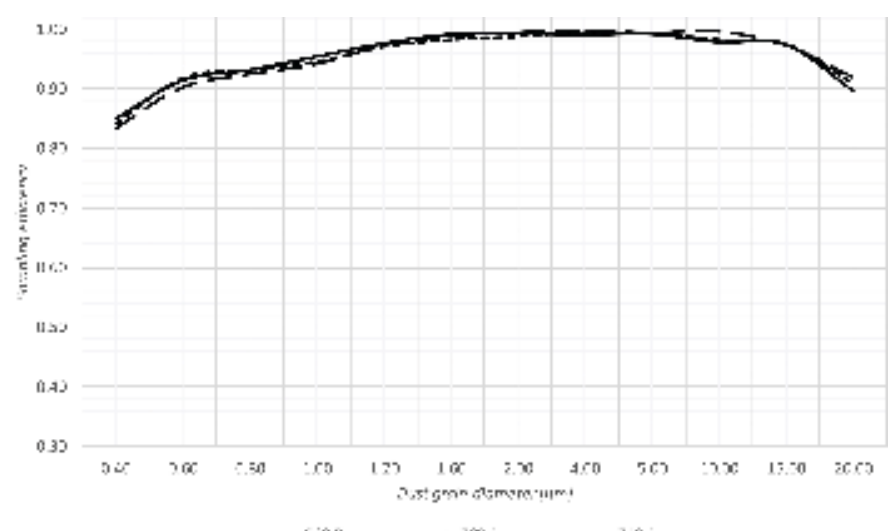

Fig. 8 Influence of daily pressure variations of the instrument sampling efficiency $\left(\mathrm{T}_{\mathrm{a}}=230 \mathrm{~K}, \mathrm{~T}_{\mathrm{i}}=253 \mathrm{~K}\right)$

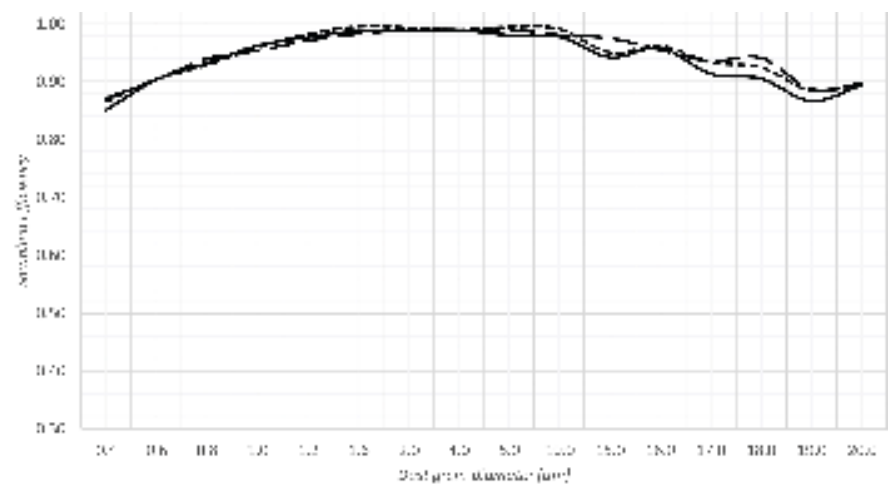

Fig. 9 Influence of seasonal atmospheric pressure on sampling efficiency $\left(\mathrm{T}_{\mathrm{a}}=190 \mathrm{~K}, \mathrm{~T}_{\mathrm{i}}=253 \mathrm{~K}\right)$

\subsubsection{Influence of atmospheric temperature}

Ambient temperature has a small effect on sampling efficiency. Indeed, the fluid rapidly reaches thermal equilibrium with the instrument, whose temperature is imposed by its position under the spacecraft thermal cover, so both fluid and dust grains velocity are not influenced by ambient temperature and sampling efficiency also shows small variations (always smaller than $7 \%$, see Fig. 10). 


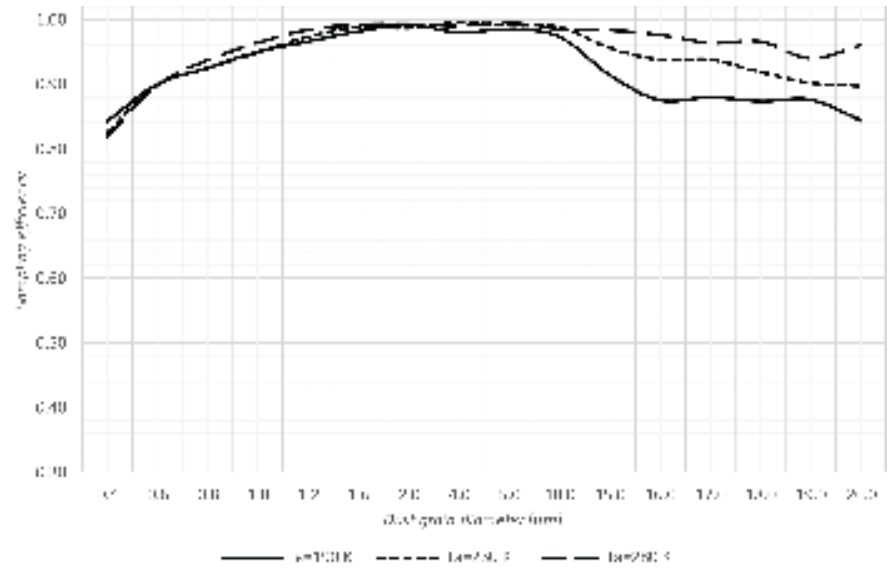

Fig. 10 Influence of atmospheric temperature on sampling efficiency $\left(\mathrm{p}_{\mathrm{a}}=600 \mathrm{~Pa}, \mathrm{~T}_{\mathrm{i}}=313 \mathrm{~K}\right)$

\subsection{Compressibility of the flow}

Dust grains velocity was an important design parameter, given that its value in the sampling section of the instrument has to be low enough for the electronics to be able to detect the grains. In MicroMED's sampling section, dust grains speed never exceeds $46 \mathrm{~m} / \mathrm{s}$, which is an acceptable value for the correct functioning of the instrument. In the sampling section, fluid speed is significantly lower than dust grains speed. This is due to the expansion the fluid experiences once it exits the inlet duct, slowing its velocity. However, the influence of this expansion on dust grains motion is small. Indeed, despite their trajectory may be slightly affected, most of the grains keep the same velocity reached in the final section of the inlet duct. After the gap, fluid speed strongly increases. Values as high as $150 \mathrm{~m} / \mathrm{s}$ are reached in the outlet section. Despite Mach number is under 0.3 for most cases and never exceeds 0.45, there is clear compressibility, favored by the extremely low pressure and density of Mars atmosphere. Moreover, instrument temperature is in most cases very different from the atmosphere's one. Therefore, sucked fluid experiences a sudden temperature increase reducing its density. Given these aspects, the original assumption of uncompressible flow was abandoned, and flow field was considered compressible.

\subsection{Particle tracking}

During CFD simulations, we track the dust grains flowing through the instrument. Fluent allows to know every particles' position while moving along MicroMED, and this feature was used to develop positional histograms for dust grains, in order to understand whether particles cross the sampling spot and, if they do, where they cross it. This is an important aspect given that the laser light is more powerful in the inner section of the sampling spot allowing an easy detection even for smaller particles (that have small reflection, meaning weaker signal) as well as a reduction of errors in the determination of size. Therefore, the exact position where the grains cross the sampling volume is a key aspect and is thus treated as a design parameter. This information can be used to describe the quality of the Breadboard fluid dynamics and helped in the development of the updated design. Fig. 11 and 12 show positional histograms for two particle dimensions in the sampling range $(2$ and $10 \mu \mathrm{m}$ in diameter, respectively). Small particles can be deflected outside the illuminated spot (whose border is highlighted by a vertical line in Fig. 11) but their distribution is peaked inside the sampling spot. The same behavior is obtained for the $10 \mu \mathrm{m}$ particles, meaning that the fluid dynamic design is able to make dust grains converge to the sampling spot for such grain dimensions. In both cases, velocity histograms are peaked around the highest value of velocity suggesting that most of the dust grains flow through the duct while being in the inner rings of the conduct where fluid speed is higher (Fig. 13-14). Bigger grains are detected with smaller efficiency not because they do not converge (they never exceed a distance of 300 $\mu \mathrm{m}$ from the center of the sampling spot, hence well inside the limit) but because they tend to hit the inlet walls, getting stuck.

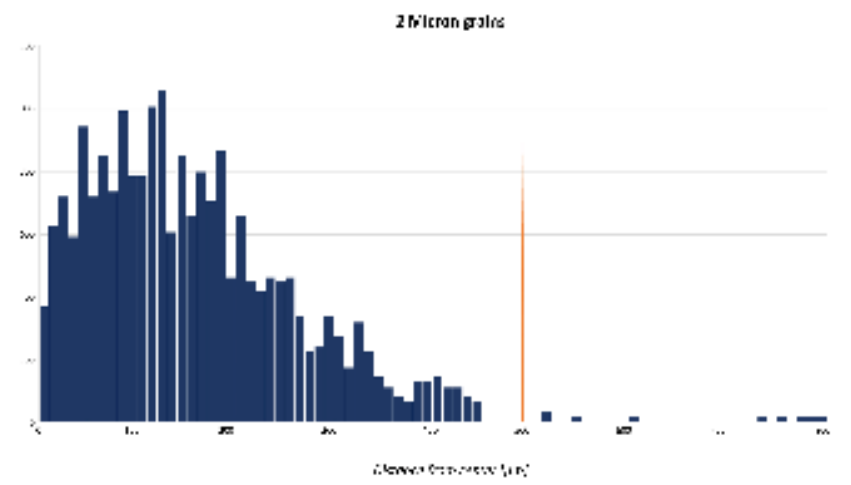

Fig. 11 Example of position histogram for $2 \mu \mathrm{m}$ grains (the vertical line represents the sampling spot border) 


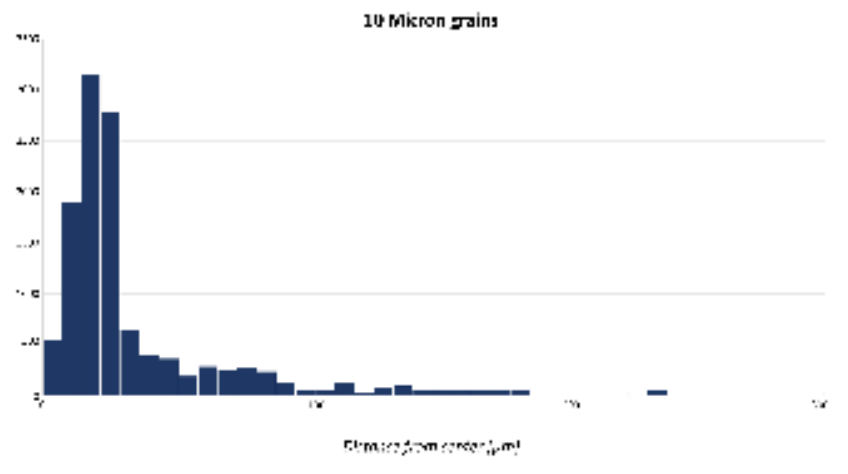

Fig. 12 Example of position histogram for $10 \mu \mathrm{m}$ grains

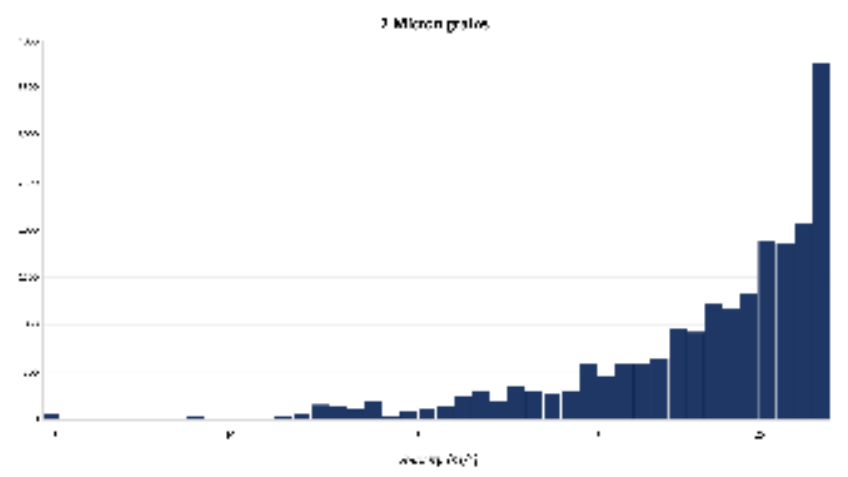

Fig. 13 Example of velocity histogram for $2 \mu \mathrm{m}$ grains

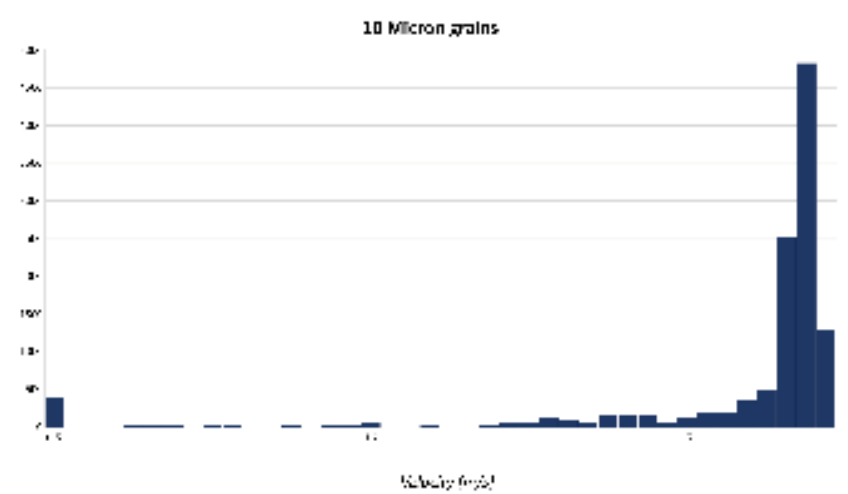

Fig. 14 Example of velocity histogram for $10 \mu \mathrm{m}$ grains

\subsection{Optimum operating conditions}

Our simulations show that the optimum operating conditions can be obtained with a $300 \mathrm{~Pa}$ pressure difference between MicroMED's inlet and outlet. This condition provides good sampling efficiency when the instrument is cold, avoiding drops in the sampling efficiency of large (16-20 $\mu \mathrm{m}$ diameter) dust grains, without altering the instrument ability to detect smaller ones. Indeed, a $300 \mathrm{~Pa} \Delta \mathrm{p}$ also guarantees sampling efficiency values over $85 \%$ (most times over $90 \%$ ) for every dust grain diameter in the $0.4-20 \mu \mathrm{m}$ range when the instrument is hot, representing a good testing condition regardless of the values assumed by environmental parameters. Anyway, MicroMED's optimum testing condition is still under debate, since the fluid dynamic design was updated in order to improve the detection of bigger grains.

\subsection{Volumetric Flow Rate}

Volumetric flow rate measurement at the inlet section is also needed to obtain the dust grain concentration value in Mars atmosphere, allowing a comparison between the number of detected dust grains and the volume of fluid sucked. Variations of atmospheric pressure, atmospheric temperature, instrument temperature all have effects on volumetric flow rate.

As in the case of sampling efficiency, the most influential parameter is instrument temperature. Volumetric flow rate values vary up to $30 \%$ between "cold" and "hot" instrument runs (see Fig. 15). It is also possible to see a gradual increase of volumetric flow rate along the instrument related to the compressibility of the flow.

Ambient temperature also has an influence but only at the inlet section (variations up to $10 \%$ ), since the fluid, once inside the instrument, is only influenced by instrument temperature because thermal equilibrium is reached (see Fig. 16).

Volumetric flow rate experiences 3-8\% variations due to daily pressure. Percentage wise, seasonal pressure has a bigger influence but only the flow rate value at the outlet section is affected, with average volumetric flow rate variations of about $7-8 \%$ with peaks up to $16 \%$ (Fig. 17).

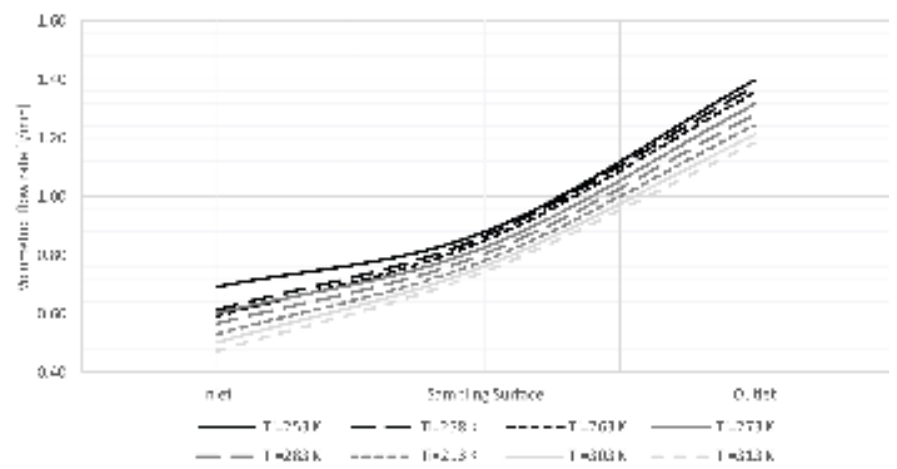

Fig. 15 Influence of instrument temperature on the instrument volumetric flow rate $\left(\mathrm{P}_{\mathrm{a}}=600 \mathrm{~Pa}, \mathrm{~T}_{\mathrm{a}}=250 \mathrm{~K}\right)$ 


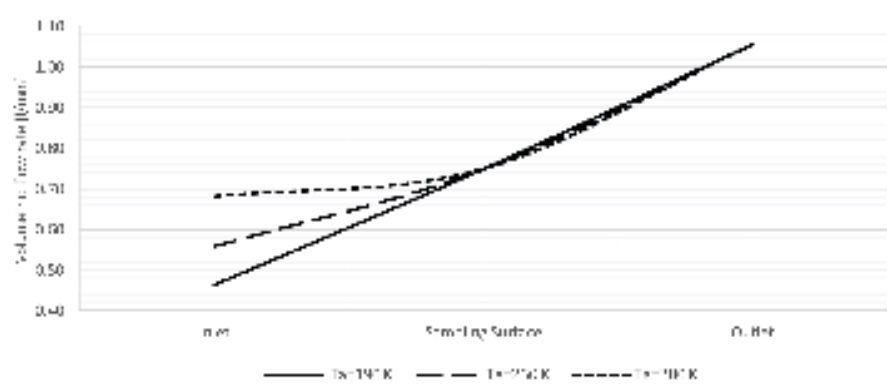

Fig. 16 Influence of atmospheric temperature on the instrument volumetric flow rate $\left(\mathrm{P}_{\mathrm{a}}=600 \mathrm{~Pa}, \mathrm{~T}_{\mathrm{i}}=253 \mathrm{~K}\right)$

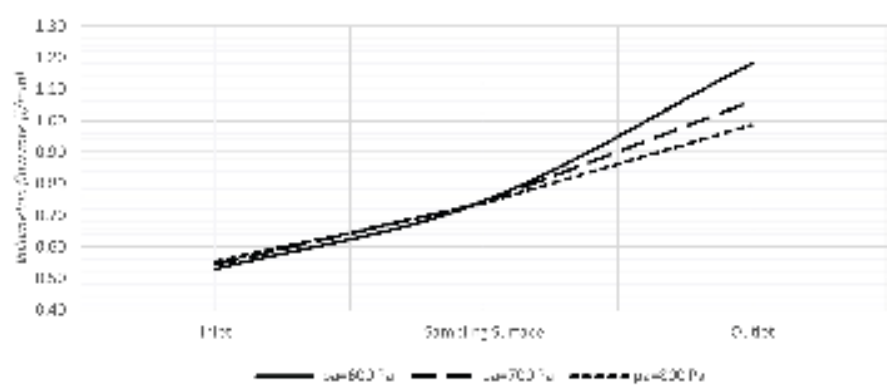

Fig. 17 Influence of atmospheric pressure on the instrument volumetric flow rate $\left(\mathrm{T}_{\mathrm{a}}=280 \mathrm{~K}, \mathrm{~T}_{\mathrm{i}}=313 \mathrm{~K}\right)$

\subsection{Behavior in windy conditions}

MicroMED's Elegant Breadboard behavior in presence of wind was studied by means of CFD simulations and results have been compared with data obtained from tests performed in Capodimonte and at the Planetary Environment Facilities (AWTSII) at Aarhus University in Aarhus, Denmark [22]. The instrument showed good performances for wind speed up to $7 \mathrm{~m} / \mathrm{s}$. For each wind speed the instrument can only detect dust grains under a threshold value that gets smaller with the increase of wind velocity. The analysis shows CFD simulations strongly underestimate the instrument ability to detect particles in windy environments. Fig. 18 shows that, according to CFD, the instrument should not be able to detect large dust grains even when wind speed is only $2 \mathrm{~m} / \mathrm{s}$, in contrast to what experienced during tests at AWTSII in Aarhus. This is probably due to the fact that Fluent considers the particles as spherical. Unsteady effects due to non-uniformity of the particles shape are not considered and the grains are simulated as more steady than they really are. CFD results can thus be considered as conservative; an improvement of the model that simulate the effect of non-sphericity of dust grains is under development.

Overall, the analysis in a windy environment will be upgraded. A more detailed analysis of the instrument's behavior in windy conditions and an analysis of the newly updated design in presence of wind is forthcoming. It is possible to see in Fig. 18 that, according to preliminary results, the new updated design improves the instrument ability to detect particles in windy environments while working at lower rpm speeds. However, there are still issues when it comes to correctly detect large dust grains when wind speed rises.

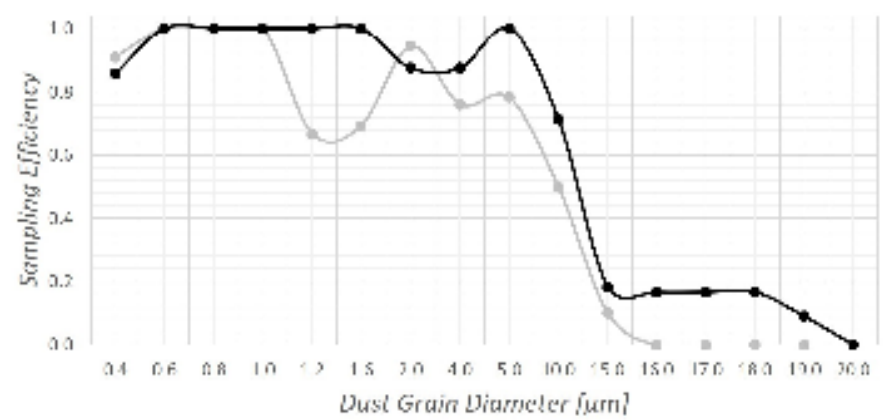

Fig. 18 Comparison between the behavior in windy environment $(\mathrm{V}=2 \mathrm{~m} / \mathrm{s})$ of the Elegant Breadboard (GREY) and the newly updated version (BLACK) of MicroMED $\left(\mathrm{p}_{\mathrm{a}}=600 \mathrm{~Pa}, \mathrm{~T}_{\mathrm{a}}=280 \mathrm{~K}, \mathrm{~T}_{\mathrm{i}}=253 \mathrm{~K}\right)$

\section{CONCLUSIONS}

MicroMED is an optical particle counter conceived to measure both abundance and size distribution of dust grains embedded in Martian atmosphere. In order to optimize the efficiency of the instrument, a CFD analysis of the instrument's Elegant Breadboard was performed and its theoretical results have been discussed, focusing on the achievable sampling efficiency and the required volumetric flow rate.

In this report we show that instrument temperature is the most influential parameter on sampling efficiency. Its effect is different depending on particles size. Dust grains of small dimensions (diameter $<1 \mu \mathrm{m}$ ) are better detected when instrument temperature is low, while the detection of larger grains (diameter $>15 \mu \mathrm{m}$ ) is more efficient for high instrument temperatures. Grains of intermediate dimensions (diameter between 1 and $15 \mu \mathrm{m}$ ) are always well detected. The instrument ability to detect large dust grains may experience a drop when the instrument is cold, a condition that highlighted the need for a geometry 
upgrade. An updated geometry has in fact been designed and its preliminary results appear positive.

Ambient pressure values (both seasonal and daily values) have small effect on the instrument sampling efficiency. Therefore, an absolute pressure sensor was not deemed necessary on MicroMED. The only pressure sensor present in the instrument is a differential pressure sensor needed to evaluate the pressure difference generated by the pump which triggers the suction of fluid.

Ambient temperature also has limited influence on MicroMED's efficiency as the fluid rapidly thermalizes with the instrument, highlighting the dominant role instrument temperature has on its functioning.

Simulation allowed to verify the flow is compressible.

Particles tracking showed the instrument design works efficiently, conveying dust grains toward the inner section of the sampling spot where laser light is more intense, improving the instrument chances to detect diffracted light.

The optimum operating conditions was found to be the one related to a $\Delta p$ generated by the pump of $300 \mathrm{~Pa}$ with respect to Martian atmospheric pressure. This condition optimizes the instrument sampling efficiency for the entire sampling range.

Volumetric flow rate is mainly influenced by instrument temperature, similarly to what was found for sampling efficiency. Pressure and ambient temperature have smaller effects.

The developed CFD model underestimates the instrument ability to detect dust grains in windy conditions, probably because the model only simulates spherical particles. An improvement of the model considering non-sphericity of grains is under development. The aforementioned updated design seems to improve MicroMED's efficiency also in presence of wind.

CFD analysis will be enhanced, as MicroMED's behavior in windy conditions has to be studied in more detail and the analysis on the newly developed design has to confirm preliminary results obtained.

\section{ACKNOWLEDGEMENTS}

This work has been supported by ASI (contract's grant number: 2016/41/H.0). The development of the instrument was funded and coordinated by ASI under the leadership of INAF-Naples, Italy. The data used in this paper can be accessed upon personal request to the first author (giuseppe.mongelluzzo@inaf.it).

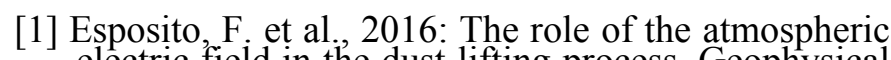
electric field in the dust-lifting process, Geophysical Research Letters, Volume 43, Issue 10, pp. 55015508

[2] Harrison, G. et al., 2016: Applications of Electrified Dust and Dust Devil Electrodynamics to Martian Atmospheric Electricity, Space Science Reviews, Volume 203, Issue 1-4, pp. 299-345.

[3] Franzese, G. et al., 2018: Electric properties of dust devils, Earth and Planetary Science Letters, Volume 493, 1. July 2018, Pages 71-81, DOI: https://doi.org/10.1016/j.eps1.2018.04.023

[4] Esposito, F. et al., 2011: MEDUSA: Observation of atmospheric dust and water vapor close to the surface of Mars, International Journal of Mars Science and Exploration, Vol. 6, p. 1-12.

[5] Colangeli, L. et al., 2009: MEDUSA: The ExoMars experiment for in-situ monitoring of dust and water vapour, Planetary and Space Science, Volume 57, Issue 8, p. 1043-1049.

[6] Esposito, F. et al., 2018: The DREAMS Experiment Onboard the Schiaparelli Module of the ExoMars 2016 Mission: Design, Performances and Expected Results, Space Science Reviews (2018), 214:103. DOI: https://doi.org/10.1007/s11214-018-0535-0

[7] Bettanini, C. et al., 2014: The DREAMS experiment on the ExoMars 2016 mission for the study of Martian environment during the dust storm season, IEEE Metrology for Aerospace (MetroAeroSpace), 2014, p. 167-173.

[8] Bettanini, C. et al., 2018: The DREAMS experiment flown on the ExoMars 2016 mission for the study of Martian environment during the dust storm season, Measurement, Volume 122, July 2018, Pages 484493 ,

DOİ:https://doi.org/10.1016/j.measurement.2018.01. 019

[9] Colangeli, L. et al., 2007: GIADA: The Grain Impact Analyser and Dust Accumulator for the Rosetta space mission, Advances in Space Research, Volume 39, Issue 3, p. 446-450.

[10] Rotundi, A. et al., 2015: Dust measurements in the coma of comet 67P/Churyumov-Gerasimenko inbound to the sun, Science, 347 , Issue 6220, article id. aaa3905.

[11] Della Corte, V. et al., 2016: 67P/C-G inner coma dust properties from 2.2 au inbound to 2.0 auoutbound to the Sun, Monthly Notices of the Royal Astronomical Society, Volume 462, Issue Suppl 1, 16 November 2016, Pages S210-S219, DOI: https://doi.org/10.1093/mnras/stw2529'

[12], Esposito, F. et al., 2002: Physical aspect of an "impact sensor" for the detection of cometary dust momentum onboard the "Rosetta" space mission, Advances in Space Research, Volume 29, Issue 8,

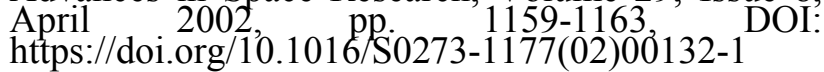

[13] Mongelluzzo, G. et al., 2018: Optimization of the fluid dynamic design of the Dust Suite-MicroMED sensor for the ExoMars 2020 mission, IEEE Metrology for Aerospace (MetroAeroSpace) 2018, p. 134-139

[14] Scaccabarozzi, D. et al., 2018: MicroMED, design of a particle analyzer for Mars, Measurement, DOI: 10.1016/j.measurement.2017.12.04

[15] Mars Climate Database v5.3, http://www.mars.lmd.jussieu.fr/mcd python/

\section{REFERENCES}


[16] Zurek et al., 1992, Dynamics of the atmosphere of Mars, Ch. 26, pp. 835-933 in Mars, The University of Arizona Press.

[17] Gomez-Elvira J., Armiens C., Carrasco I. et al., 2014, Curiosity's rover environmental monitoring station: Overview of the first 100 sols. J. Geophys. Res. Planets, 119, 1680-1688, doi:10.1002/2013JE004576.

[18] Anderson, J.D. et al., 1989: Hypersonic and HighTemperature Gas Dynamics (Second Edition), AIAA Education Series, p. 564-567

[19] Allen, M.D., Raabe, O.G., Re-Evaluation of Millikan's Oil' Drop Data for the Motion of Small Particles in Air - Journal of Aerosol Science, Vol. 13, N. 6, p. 537-547, 1982

[20] Hutchins, D.K., Harper, M.H., Felder, R.L., Slip Correction Measurements for Solid Spherical Particles by Modulated Dynamic Light Scattering -
Aerosol Science and Technology, Vol. 22, Issue 2, p. 202, 1995

[21] Kim, J.H. et al., Slip Correction Measurements of Certified PSL Nanoparticles Using a Nanometer Differential Mobility Analyzer (Nano-DMA) for Knudsen Number From 0.5 to 83, Journal of Research of the National Institute of Standards and Technology, Volume 110, Number 1, JanuaryFebruary 2005, p. 31-54

[22] Holstein-Rathlou et al., 2014, An Environmental Wind Tunnel Facility for Testing Meteorological Sensor Systems, American Meteorological Society, 31,447 